\title{
Analyzing bit error rate of relay sensors selection in wireless cooperative communication systems
}

\author{
Ahmed Allawy Alawady ${ }^{1}$, Ahmed Alkhayyat ${ }^{2}$, Mohammed Ahmed Jubair ${ }^{3}$, Mustafa Hamid Hassan ${ }^{4}$, \\ Salama A. Mostafa ${ }^{5}$ \\ 1,2 Department of Computer Technical Engineering, College of Technical Engineering, The Islamic University, 54001 \\ Najaf, Iraq \\ 3,4,5 Faculty of Computer Science and Information Technology, Universiti Tun Hussein Onn Malaysia, Malaysia
}

\begin{tabular}{l} 
Article Info \\
\hline Article history: \\
Received Apr 30, 2020 \\
Revised May 14, 2020 \\
Accepted Jun 27, 2020 \\
\hline
\end{tabular}

\section{Keywords:}

Bit error rate

Cooperative communication

IoT

WBAN

\begin{abstract}
Cooperative communication systems, which make use of the intermediate relays between the transmitter and the receiver, have been employed as an effective technique to combat the channel fading and to enhance system performance. Cooperative systems have some drawbacks such as high latency and may diversity order not guaranteed. To alleviate the negative effects of these factors, the relay selection protocol is employed in cooperative communication systems to increase overall cooperative system performance. Relay selection in the cooperative systems enables the source to cooperate with the single relay node rather than multiple relay nodes which guaranteed the diversity order.
\end{abstract}

This is an open access article under the CC BY-SA license.

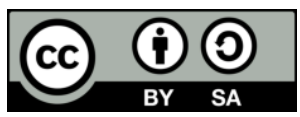

Corresponding Author:

Ahmed Allawy Alawady,

Department of Computer Technical Engineering,

College of Technical Engineering,

The Islamic University,

54001 Najaf, Iraq.

Email: ahmed.allawy2001@gmail.com

\section{INTRODUCTION}

Cooperative networks (CNs) have been grown and gain much attention recently as a new model of the communication system that makes intermediate nodes help the source to retransmitted its data if the source to destination link (SDL) failed due to error propagation or the relay nodes retransmit the source data in addition transmitted source data and the destination combine them to achieve diversity gain. To achieve the diversity gain through cooperation, the source broadcast the data to the relay node and the destination (exploiting the broadcast nature of the wireless medium in $\mathrm{CNs}$ ), and either the relay nodes process the data that received from the source before retransmitting to the destination, or the relay nodes doesn't process the data but directly retransmit to the destination. The relay nodes can process the data such that estimation, demodulation and forward, decoding and re-encoding, etc.

Recently, inclusive work on CNs have been inspected as the relay nodes repeat the data to the destination and it receives the data from the relay nodes only has been considered in [1-3], or the destination receive the data from the source and relay nodes has been considered in [4-7]. Generally, if the relay nodes process the data before retransmitting that mean the relay nodes within the range of the source and the destination and such retransmitting, it can cause a reduction in the spectral efficiency (degree of the freedom of the channels). Loosely speaking, the cooperation is to achieve diversity gain but multiplexing gain may not 
if the cooperation system not designs properly. Hence it is important to evaluate their performance in the term of diversity-multiplexing trade-off [8].

Since, retransmission the data by the relay nodes to the destination, it is inherently reducing the spectral efficiency. There are several techniques have been explored in the literature to overcome the above problems through distributed space-time coding $[9,10]$, relay nodes work on full-duplex, it is mean the relay node can receive and transmit at same time [11], dynamic allocation of the time slots [12], best relay selection $[13,14]$. In the practical, the implementation of distributed space code require to setup multiple antennas at the mobile set and this not practical for small devices, full-duplex cooperation required the relay to cancel its self-interference from the received signal, but this not robust in the low-cost radio devices, dynamic allocation required overhead and global information, relay selection is a simplistic way and can achieve spectral efficiency as well as diversity gain.

The best relay selection (BRS) can generally be categorized into two classes: the reactive BRS [15-17], and proactive BRS [18-20]. The reactive BRS protocol prompts the relay selection process after the source broadcasting the data to the destination and relay nodes. However, the proactive BRS protocol in selecting the relay node before the source broadcasting (transmitting). The disadvantage of the proactive BRS is that relay node retransmits what was transmitted by the source to the destination even when the direct transmission is sufficient such a disadvantage reduce the spectral efficiency. Hence designing an efficient and robust BRS can achieve better spectral efficiency.

Internet of Things (IoT) has been an ever-growing ecosystem that integrates hardware, computing devices, physical objects, software, and animals or people over a network enabling them to interact, communicate, collect and exchange data. There are different major types of IoT services, from the industrial applications to eHealth care applications. In this work, the smart wearable/implants sensors, machine devices attached on or inside a human body for monitoring in a hospital digital healthcare system are concerned, to collect data about the health status of a person for a heartbeat, blood pressure, glucose level, etc., through the sensors on the wearable technologies [21-23]. Wireless body area networks which it is abbreviated as WBAN can be practically utilized for delivering information related to the human body for a master node or central computer [24, 25], in which it has the ability for being observed, saved as well as examined theoretically. Reliability is a major issue of the WBAN, for this reason, cooperative communication is considered to provide reliable and efficient delivery of the information [26, 27].

The contributions of this paper are summarized as follow:

a. We propose a relay selection protocol that selects the best relay node base on link quality from the source to relay and from the relay to the destination that can gain the cooperation system better performance.

b. We assume the relay process the data before retransmitting to the destination, where the process type is decoding and re-encoding again that offer extra coding gain to the cooperation system and the spectral efficiency.

c. We reveal the proposed protocol can reduce the bit error rate compared to classical cooperation mode (cooperation without selection protocol) and previous work.

A new architecture of an IoT health-based paradigm is shown in the Figure 1 which can be divided into four layers. The WBSN (wireless body sensors network) layer (layer\#1), in this layer, sensors might be attached directly to the human body or sewed into the fabric (wearable sensors), or implanted inside the human body. Such sensors can be EEG, ECG, or EMG, etc. The data recorded via sensors are transmitted to the master node $(\mathrm{MN})$ via wireless 802.15 .6 standard, then $\mathrm{MN}$ transfer what were transmitted by the sensors to the next tier over one of the wireless technology or cables.

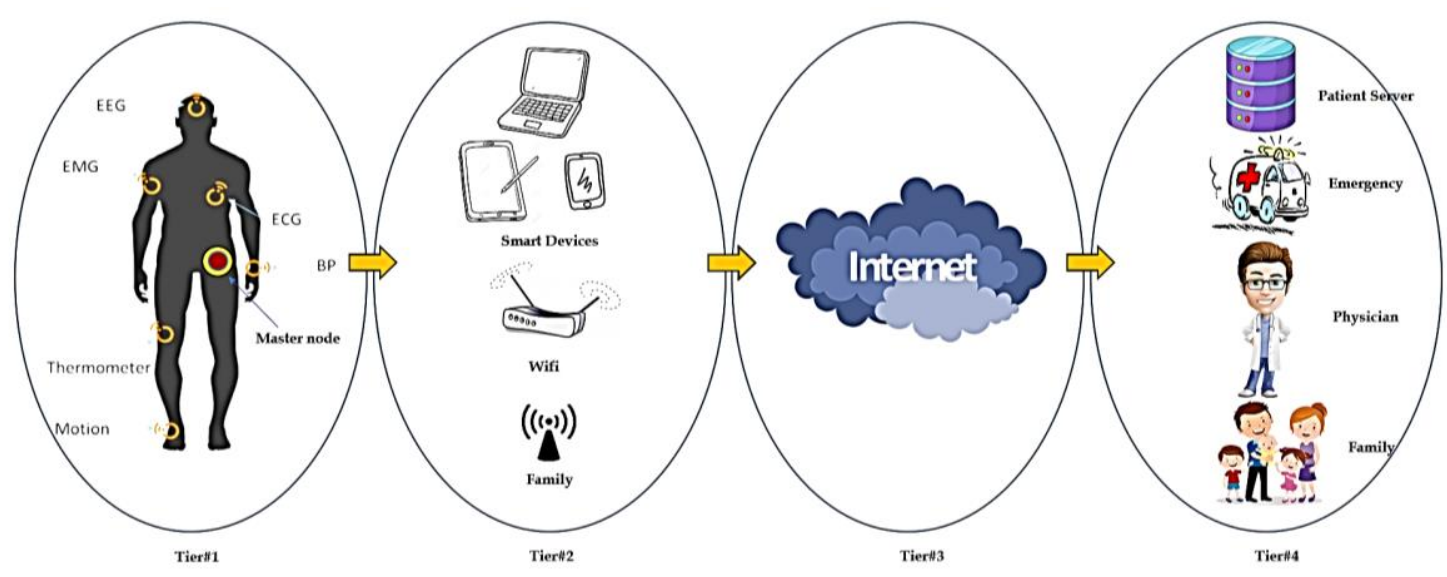

Figure 1. An example of the WBSN in IoT-based health networks 
Smart interface (layer\#2), in this layer, smart devices are utilized, (i.e., smartphone, laptop or tablet). In this layer, data are inspected and analysed, then the data transferred to layer 3 over one of the selected wireless communication technologies (i.e., Bluetooth, Wi-Fi or cellular Base-station). Layer 2 represents the bridge tier that connects the WBSN to the infrastructure internet, and some time located within WBSN area. Infrastructure internet layer (layer\#3), this level bridge the gap between the layer\#2 and layer\#4 via exiting communication technology. Care-Services layer (layer\#4), in this layer, the received data patient server, the Patient server: in this layer, the data are stored, analyzed and forwarded to the suitable service, such as emergency, physician, or family. The rest of the paper is organized as follow: We provide a channel formulation of decode and re-encode of CNs in section 2. In section 3, we present the details and the main idea of a proposed relay selection protocol, in the sequel, we evaluate the spectral efficiency, bit error rate, diversity order and outage probabilities of the proposed relay selection protocol in the subsections', 3.1, 3.2 and 3.3. Analysis and performance results are addressed in section 4. And finally, section 6 draws the conclusion and future work.

\section{RELAY SELECTION FOR PROPOSED ARCHITECTURE}

\subsection{Inter-node SNR modelling}

In this section, the channel formulation of the decode and forward cooperation is described. In this work, the cooperation takes place over the two-time slot, first-time slot sensor broadcast the data over a wireless channel using 802.15.6 for second-tier and third-tier, then at second time slot, the D2D users re-forward the received data to the third tier over $802.11 \mathrm{~b}$. In the first time slot, the received signals at the destination $(\mathcal{Y} \mathrm{t} 1-\mathrm{t} 3)$ and relay $(\mathcal{Y} \mathrm{t} 2-\mathrm{t} 3)$ are given as:

$$
\begin{aligned}
& y_{t 1-t 3}=\sqrt{P_{b}} h_{t 1-t 3} x+\eta_{t 1-t 3} \\
& y_{t 1-t 2}=\sqrt{P_{b}} h_{t 2-t 3} x+\eta_{t 2-t 3}
\end{aligned}
$$

The $h_{t 1-t 3}, h_{t 1-t 2}$ are the fading coefficients for corresponding tier\#1 to tier\#3 and tier\#1 to tier\#2 links, respectively; $x$ is the modulated signal transmitted from the source, and $\eta_{t 1-t 3}, \eta_{t 2-t 3}$ are the complex white Gaussian noise with zero mean and unit variance for corresponding tier\#1 to tier\#3 and tier\#1 to tier\#2 links, respectively. In the second time slot, the signal received at the destination ( $\mathcal{Y}$ rd) from the relay is given as:

$$
\mathcal{Y}_{t 2-t 3}=\sqrt{P_{m}} h_{t 2-t 3} \hat{x}+\eta_{t 2-t 3} \text {. }
$$

The $\mathrm{h}_{t 2-t 3}$ is the fading coefficient for corresponding tier\#2 to tier\#3 links, $\hat{x}$ is the modulated signal transmitted from the relay to the destination, and $\eta_{t 2-t 3}$ is the complex white Gaussian noise with zero mean and the unit variance for corresponding tier\#2 to tier\#3 links. In what follows, the instantaneously received signal-to-noise ratio of the three links, tier\#1 to tier\#3 and tier\#1 to tier\#2 and tier\#1 to tier\#2 links, are given as:

$$
\begin{aligned}
& \gamma_{t 1-t 3}=\frac{P_{b}}{N_{o}}\left|h_{t 1-t 3}\right|^{2} . \\
& \gamma_{t 1-t 2}=\frac{P_{m}}{N_{o}}\left|h_{t 2-t 3}\right|^{2} . \\
& \gamma_{D F}=\frac{P_{b}}{N_{o}}\left|h_{t 2-t 3}\right|^{2}+\frac{P_{m}}{N_{o}}\left|h_{t 2-t 3}\right|^{2} .
\end{aligned}
$$

\subsection{Relay selection}

In this section, the proposed best D2D users selection (BD2DS) algorithm is described. The proposed BD2DS algorithm operation is modified version of the traditional automatic repeat request (ARQ) algorithm, if the tier\#3 device transmits a negative ACK to the sensors (tier\#1 device) indicating the failure of the reception, the tier\#2 device retransmits the lost signal tier\#3 device, then tier\#3 device sum-u the signals via maximal ratio combing (MRC). Accordingly, The BD2DS algorithm in this paper can be summarized as follow; 1) the tier\#3 device admit a direct transmission signal' (tier\#1 to tier\#3) if the link quality of tier\#1 to tier\#3 greater than the links quality of tier\#1 to tier\#2 and tier\#2 to tier\#3, 2) the best D2D users' do retransmission if the link quality of tier\#1 to tier\#3 less than the quality of the links of the tier\#1 to tier\#2 and tier\#2 to tier\#3. Thus, the BD2DS selection methodology can be mathematically modelled as: 


$$
\mu=\beta_{t 1-t 3}>\beta_{\max }
$$

Let $\beta_{t 1-t 3}$ be a common channel coefficient representing the channel between any given two nodes. $\beta_{t 1-t 3}$ is modelled as zero-mean complex Gaussian random variables with variance $\sigma_{t 1-t 3}$. The $\beta_{\min -\max }=$ $\min \left\{\max \left\{\beta_{1}, \beta_{2}, \ldots ., \beta_{k}\right\}\right\}, \quad \beta_{k}=\max \left(\left(\sigma_{(t 1-t 3)_{k}}^{2} / d_{(t 1-t 3)_{k}}^{\alpha}\right),\left(\sigma_{(t 2)_{k} d}^{2} / d_{(t 2) d}^{\alpha}\right)\right)$, In which $\mathrm{k}=1,2, \ldots ., \mathrm{L}$ is the number of D2D users in the tier\#2. Based on the event given in (7), the proposed BD2DS) algorithm can be described as follows; after the sensors broadcasting the data to the destination and D2D users. In the second time slot, the tier\#3 device checks the criteria given in (7), and if the criteria $\beta_{t 1-t 3}>\beta_{\min -\max }$ is satisfied the tier\#3 device resolves the transmitted data and transmits positive ACK, otherwise the tier\#3 device transmits negative ACK. In the latter case, the best D2D users node re-transmits its decoded re-encoded data. The tier\#3 device is assumed knows the quality of the links of the tier\#1 to tier\#2 and tier\#2 to tier\#3 of all D2D users.

\subsection{Bit error rate formation of the proposed method}

In this section, it is shown the BD2DS algorithm has diversity order is 2 , in which single relay has been chosen within the relay region. The general definition of the error probability between two nodes $(i ; j)$ given as [28]:

$$
P_{e}=Q\left(\sqrt{A\left|h_{i, j}\right|^{2}}\right) \leq \frac{1}{\pi} \int_{0}^{\frac{(M-1) \pi}{M}} \exp \left(-A A_{\theta}\left|h_{i, j}\right|^{2}\right) d \theta
$$

In which the $A=k_{p s k} P_{b}$, kpsk is $(\sin (\pi / \mathrm{M})) /$ No $M$ is the modulation order, and $A_{\theta}=\left(1 / \sin ^{2}(\theta)\right)$. The expression of error probability of a decode and forward algorithm consists of two parts; 1) the source broadcasts the data to the destination and relays, but the relay does not forward to the destination the data that received from the source since the destination send a positive ACK to relay, 2) the destination receives the data from source and relays since it sends a negative ACK to relay. Therefore, considering these cases, the error probability of the DF algorithm is given as [23]:

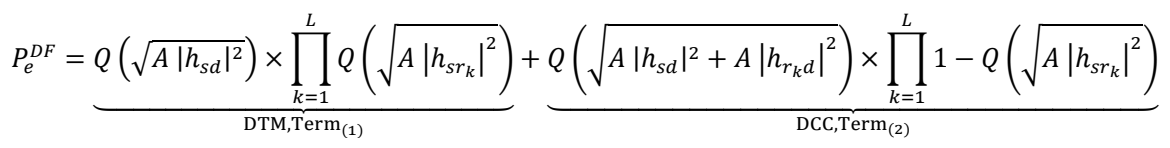

İn which $1-Q\left(\sqrt{A\left|h_{s r_{k}}\right|^{2}}\right)$ is error-free of tier\#1 to tier\#2 links. The probability of error of the DF algorithm can be upper bounded by removing the negative term and setting $L=1$ we rewrite (9) as:

$$
P_{e}^{D F}=\underbrace{\underbrace{\frac{1}{\pi^{2}} \int_{0}^{\frac{(M-1) \pi}{M}} \exp \left(-A A_{\theta}\left|h_{s d}\right|^{2}\right)}_{0} \exp \left(-A A_{\theta}\left|h_{s r}\right|^{2}\right) d \theta d \theta}_{\text {broadcasting phase-1 }}+\underbrace{\frac{1}{\pi} \int_{0}^{\frac{(M-1) \pi}{M}} \exp \left(-\left(A A_{\theta}\left|h_{s d}\right|^{2}+A A_{\theta}\left|h_{r d}\right|^{2}\right)\right) d \theta}_{\text {retransmission phase-2 }}
$$

The error probability of BD2DS algorithm can be express as:

$$
P_{e}^{D F}=P_{e}^{b p} \mathrm{P}(\mu)+P_{e}^{r p} \overline{\mathrm{P}(\mu)}
$$

The first term of (11) is comprised of two multiplied terms, the probability of error of the broadcasting phase ' $P_{e}^{b p}$, and the probability of the correct reception from the broadcasting phase ' $\mathrm{P}(\psi)$ '. The first term can be express as:

$$
P_{e}^{b p} \mathrm{P}(\mu)=\int_{0}^{\infty} P(e \mid \mu) \operatorname{Pr}\left(\mu \mid \frac{k_{s d}}{\lambda}\right) p_{I_{s d}}\left(I_{s d}\right) d I_{s d} d I_{s r}
$$

The $\operatorname{Pr}(e \mid \psi)$ is given in (10) as broadcasting phase term, $\operatorname{Pr}\left(\psi \mid I_{s d} / \lambda\right)=\left(1-\exp \left(-\left(\gamma_{1}+\gamma_{2}\right) I_{s d} /\right.\right.$ $\lambda))$ and $p_{I_{s d}}\left(I_{s d}\right)=\left(1 / \sigma_{s d}\right) \exp \left(-I_{s d} / \sigma_{s d}\right)$, we rewrite (12) as:

$$
\operatorname{Pr}(e \mid \psi)_{T e r m(1)} \operatorname{Pr}(\psi)=\frac{1}{\sigma_{s d}^{2} \pi^{2}} \int_{0}^{\infty} \exp \left(-A I_{s r}\right)\left(1-\exp \left(-\frac{I_{s d}}{\lambda}\left(\gamma_{1}+\gamma_{2}\right)\right)\right) d \theta d \theta d I_{s d} d I_{s r}
$$


Then (13) can be upper bounded by setting $\sin (\theta)=1$ and $\int_{0}^{\frac{(M-1) \pi}{M}} \int_{0}^{\frac{(M-1) \pi}{M}} d \theta d \theta=\left(\frac{(M-1)}{M}\right)^{2}[29,30]$, then we rewrite (13) as:

$$
P_{e}^{b p} \mathrm{P}(\mu) \leq \frac{\bar{m}}{\sigma_{s d}^{2}} \int_{0}^{\infty} \exp \left(-A I_{s d}-\frac{I_{s d}}{\sigma_{s d}^{2}}\right) \exp \left(-A I_{s r}\right)\left(1-\exp \left(-\frac{I_{s d}}{\lambda}\left(\gamma_{1}+\gamma_{2}\right)\right)\right) d I_{s d} d I_{s r}
$$

Solving the integration of (14) concerning Isd, Isr and substitute $\gamma_{1}$ and $\gamma_{1}$; further, SDL is assumed to be fixed during the cooperation, $\sigma_{s d}^{2}=1$. We rewrite (14) as:

$$
P_{e}^{b p} \mathrm{P}(\mu) \leq \frac{\bar{m}}{k_{p s k} A_{b} P_{b}+1}\left(A \sigma_{s r}^{2}\right)^{-1}-\bar{m}\left(1+A+\frac{1}{\lambda}\left(\sigma_{s r}^{2}+\sigma_{d r}^{2}\right)\right)^{-1}
$$

In which $\bar{m}$ is $\left(\frac{M}{M-1}\right)^{2}$

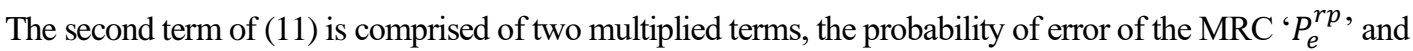
the probability of the correct reception from both relay and direct transmission ' $\mathrm{P}(\bar{\mu})$ '. can be express as:

$$
P_{e}^{r p} \overline{\mathrm{P}(\mu)}=\int_{\bar{I}}^{\prime} P(e \mid \bar{\mu}) \operatorname{Pr}(\bar{\mu} \mid \bar{I}) p_{\bar{I}}(\bar{I}) d \bar{I}
$$

In which $\bar{I}:=\left[I_{s d}, I_{s r}, I_{r d}\right] . \operatorname{Pr}(\bar{\mu} \mid \bar{I})$ is the probability of the BD2DS algorithm protocol and it is given as:

$$
\operatorname{Pr}\left(\bar{\mu} \mid I_{s d}, I_{s r}, I_{r d}\right)=\operatorname{Pr}\left(I_{s d}<\lambda I_{\max } \mid \bar{I}\right)=1-\exp \left(-\left(A A_{\theta}+\frac{1}{\sigma_{s d}^{2}}\right) \lambda I_{\max }\right)
$$

$P(e \mid \bar{\mu})$ is the second term of the $(10)$, and $p_{\bar{I}}(\bar{I})=p\left(I_{s d}\right) p\left(I_{s r}\right) p\left(I_{r d}\right)$; we rewrite (16) as:

$$
\begin{aligned}
& P_{e}^{r p} \overline{\mathrm{P}(\mu)} \leq \frac{1}{\pi} \int_{\bar{I}} \int_{0}^{\frac{(M-1)_{\pi}}{M}} \exp \left(-A A_{\theta}\left(I_{s d}+I_{r d}\right)\right)\left(1-\exp \left(-\left(A A_{\theta}+\frac{1}{\sigma_{s d}^{2}}\right) \lambda I_{\max }\right)\right) \\
& p_{I_{s d}}\left(I_{s d}\right) p_{I_{s r}}\left(I_{s r}\right) p_{I_{r d}}\left(I_{r d}\right) d \theta d I_{s d} d I_{s r} d I_{r d}
\end{aligned}
$$

Evaluate the integration concerning Isd and we take into account the upper bound assumption that is made on (14), we rewrite (18) as:

$$
P_{e}^{r p} \overline{\mathrm{P}(\mu)} \leq \ddot{m} \int_{\bar{I}}^{\prime}(A)^{-1} \exp (-A)\left(1-\exp \left(-(A+1) \lambda I_{m a x}\right)\right) p_{I_{s r}}\left(I_{s r}\right) p_{I_{r d}}\left(I_{r d}\right) d I_{s r} d I_{r d}
$$

in which $\ddot{m}$ is $M /(M-1)$; taking into account worst case state by substituting Isr=k Imax and $\operatorname{Ird}=\mathrm{k} \operatorname{Imax}$, where $\mathrm{r}$, i.e., $\operatorname{Pr}(\mathrm{e}) \mathrm{k}=0.1>\operatorname{Pr}(\mathrm{e}) \mathrm{k}=0.2>\ldots .>\operatorname{Pr}(\mathrm{e}) \mathrm{k}=0.9>\operatorname{Pr}(\mathrm{e}) \mathrm{k}=1$, then we rewrite (19) as:

$$
\begin{aligned}
& \operatorname{Pr}\left(e \mid \psi_{D C C}\right)_{T \operatorname{erm}(2)} \operatorname{Pr}\left(\psi_{D C C}\right) \leq \ddot{m}(A+1)^{-1} \int_{I_{\max }}^{\prime} \exp \left(-A \kappa I_{\max }\right) p\left(I_{\max }\right) d I_{\max }- \\
& \ddot{m}(A+1)^{-1} \int_{I_{\max }^{\prime}}^{\prime} \exp \left(-\left(A \kappa I_{\max }+\lambda A+\lambda\right) I_{\max }\right) d I_{\max }
\end{aligned}
$$

Given a moment generating function of $\operatorname{Imax}, M_{I_{\max }}($.$) [31], we can rewrite (20) as:$

$$
\operatorname{Pr}\left(e \mid \psi_{D F}\right) \operatorname{Pr}\left(\psi_{D F}\right)_{T e r m(2)} \leq \ddot{m}(A+1)^{-1}\left[M_{I_{\max }}(A \kappa)-M_{I_{\max }}((A+\lambda A+\lambda))\right]
$$

The Imax is constituted two random variables are Isr and Ird, and the moment generating function of two independent random variables is given as $\mathrm{Mx}, \mathrm{y}(\mathrm{s}, \mathrm{t})=\mathrm{Mx}(\mathrm{s}) \mathrm{My}(\mathrm{t})$, then we rewrite $(21)$ as:

$$
\begin{aligned}
& \operatorname{Pr}\left(e \mid \psi_{D F}\right) \operatorname{Pr}\left(\psi_{D F}\right)_{T e r m(2)} \leq \ddot{m}\left(k_{p s k} A_{b} P_{b}+1\right)^{-1} \omega_{\sigma}\left[\left(\frac{d_{s r}^{\beta}}{\sigma_{s r^{2}}}+F\left(P_{b}\right)\right)^{-1}\left(\frac{d_{r d}^{\beta}}{\sigma_{r d^{2}}}+F\left(P_{b}\right)\right)^{-1}-\right. \\
& \left.\left(\frac{d_{r d}^{\beta}}{\sigma_{r d^{2}}}+F\left(P_{b}, P_{m}\right)\right)^{-1}\left(\frac{d_{s r}^{\beta}}{\sigma_{s r^{2}}}+F\left(P_{b}, P_{m}\right)\right)^{-1}\right]
\end{aligned}
$$

in which $\omega_{\sigma}=\left(\left(d_{s r}^{\beta} \sigma_{r d}^{2}+d_{s r}^{\beta} \sigma_{r d}^{2}\right) / \sigma_{r d}^{2} \times \sigma_{r d}^{2}\right), \quad \mathrm{F}(\mathrm{Pm})=\mathrm{kpsk}$ Pm $\mathrm{k}$ and $F\left(P_{b}, P_{m}\right)=\left(k_{p s k} P_{m} k+\lambda\right.$ $\left.k_{p s k} P_{m} k+\lambda\right)$. Finally, the total error probability of BD2DS algorithm is obtained by adding the (15) and (23), it is given as: 


$$
\begin{aligned}
& \operatorname{Pr}(e) \leq \ddot{m}(A+1)^{-1}\left(\ddot{m}\left(A \frac{\sigma_{s r}^{2}}{d_{s r}^{\beta}}\right)^{-1}-\ddot{m}\left(1+A+\frac{1}{\lambda}\left(\frac{d_{s r}^{\beta}}{\sigma_{s r}^{2}}+\frac{d_{r d}^{\beta}}{\sigma_{r d}^{2}}\right)\right)^{-1}+\omega_{\sigma}\left[\left(\frac{d_{s r}^{\beta}}{\sigma_{s r^{2}}}+\right.\right.\right. \\
& \left.\left.\left.F\left(P_{b}\right)\right)^{-1}\left(\frac{d_{r d}^{\beta}}{\sigma_{r d^{2}}}+F\left(P_{b}\right)\right)^{-1}-\left(\frac{d_{r d}^{\beta}}{\sigma_{r d^{2}}}+F\left(P_{b}, P_{m}\right)\right)^{-1}\left(\frac{d_{s r}^{\beta}}{\sigma_{s r^{2}}}+F\left(P_{b}, P_{m}\right)\right)^{-1}\right]\right)
\end{aligned}
$$

\section{SIMULATION AND RESULTS}

It is clear that, as the selection criteria consider both distances and links quality, the cooperation probability is higher compare to the case that considering one of the channel characteristics, i.e., either distance or channel gain. Figure 2 shows the bit error rate compares to the signal-to-noise ratio (SNR), the important results appear in the Figure 2 can be summarized as follow. The bit error rate reduces once the classical cooperation employed compare to direct transmission. The bit error rate reduces more compare to classical cooperation mode in the previous case, further enhancement as selection consider the best relay node in mid-distance among the source and the destination. Selecting a node far away from the source or the destination (not in the middle location between source and destination), the performance reduces from the case as we select the node at the mid-distance among the source and the destination.

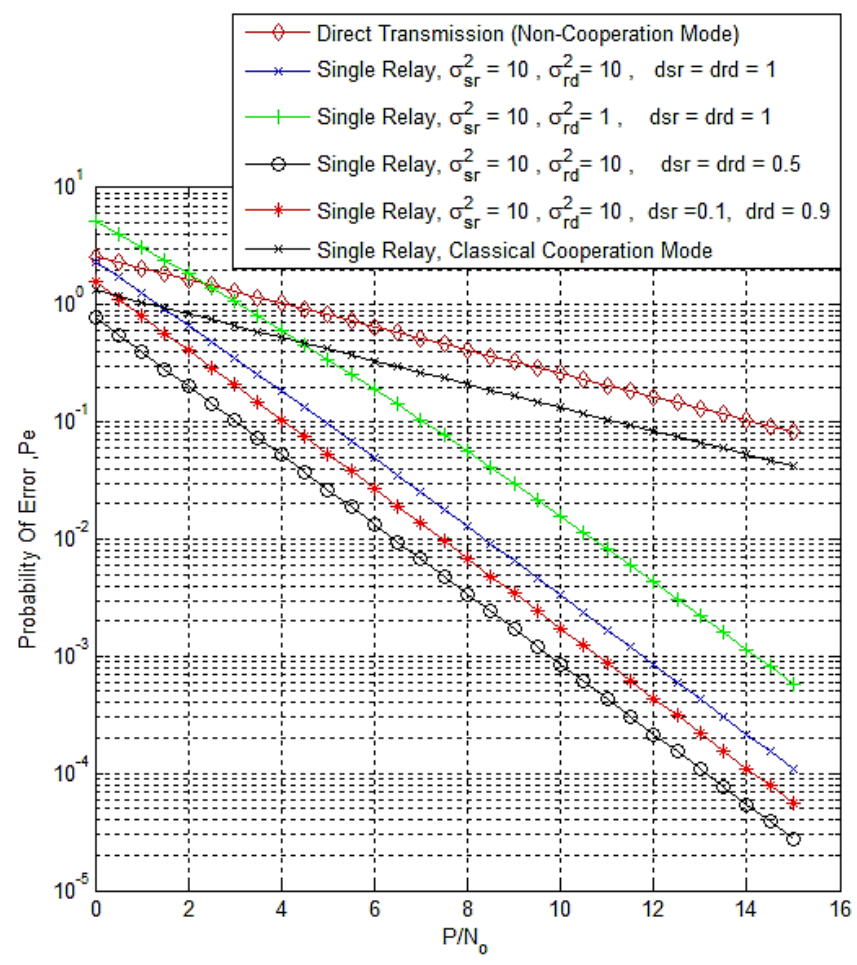

Figure 2. The bit error rate for direct transmission, with relay selection and without relay selection of cooperative mode channels gain and distances from the source to relay and from the relay to destination

\section{CONCLUSION}

In this paper, we have proposed a relay selection protocol for cooperative networks. the advantage of the cooperation and relay selection can be exploited. The relay selection criteria based on a maximum link from the source to relay and from the relay to the destination, we demonstrated that the proposed relay selection can substantially improve the spectral efficiency, bit error rate, diversity order and outage probability comparing with non-selective cooperative networks.

\section{ACKNOWLEDGEMENTS}

This research is supported by the Islamic University, Najaf, Iraq. It is also supported by Research Fund to E15501, Research Management Center, UTHM. 


\section{REFERENCES}

[1] P. Liu, Z. Tao, S. Narayanan, T. Korakis and S. S. Panwar, "CoopMAC: A Cooperative MAC for Wireless LANs," in IEEE Journal on Selected Areas in Communications, vol. 25, no. 2, pp. 340-354, 2007.

[2] R. R. Ali, S. A. Mostafa, H. Mahdin, A. Mustapha and S. S. Gunasekaran, "Incorporating the Markov Chain Model in WBSN for Improving Patients' Remote Monitoring Systems," In Recent Advances on Soft Computing and Data Mining, vol. 978, pp. 35-46, 2020.

[3] M. Khalid, Y. Wang, I. Ra and R. Sankar, "Two-Relay-Based Cooperative MAC Protocol for Wireless Ad hoc Networks," in IEEE Transactions on Vehicular Technology, vol. 60, no. 7, pp. 3361-3373, 2011.

[4] A. Alkhayyat, O. Gazi and S. B. Sadkhan, "The role of delay and connectivity in throughput reduction of cooperative decentralized wireless networks," Mathematical Problems in Engineering, vol. 2015, pp. 1-10, 2015.

[5] M. A. Jubair, S. A. Mostafa, R. C. Muniyandi, H. Mahdin, A. Mustapha, M. H. Hassan, M. A. Mahmoud, Y. A. AlJawhar, A. S. Al-Khaleefa, and A. J. Mahmood "Bat optimized link state routing protocol for energy-aware mobile ad-hoc networks," Symmetry, vol. 11, no. 11, p. 1409, 2019.

[6] A. Alkhayyat and S. B. Sadkhan, "Bandwidth efficiency analysis of cooperative communication with Reactive Relay Selection," 2018 International Conference on Engineering Technology and their Applications (IICETA), AlNajaf, pp. 77-80, 2018.

[7] A. alkhayyat, K. Al Attabi and Q. h. Abbasi, "Single Relay Selection in the Cognitive Cooperative Network: Toward Bandwidth Efficiency Improvement," 2019 4th Scientific International Conference Najaf (SICN), Al-Najef, Iraq, pp. 222-226, 2019.

[8] Lizhong Zheng and D. N. C. Tse, "Diversity and multiplexing: a fundamental tradeoff in multiple-antenna channels," in IEEE Transactions on Information Theory, vol. 49, no. 5, pp. 1073-1096, 2003.

[9] J. N. Laneman and G. W. Wornell, "Distributed space-time coded protocols for exploiting cooperative diversity in wireless networks," Global Telecommunications Conference, 2002. GLOBECOM '02. IEEE, Taipei, Taiwan, vol.1, pp. 77-81, 2002.

[10] Y. Jing and B. Hassibi, "Distributed Space-Time Coding in Wireless Relay Networks," in IEEE Transactions on Wireless Communications, vol. 5, no. 12, pp. 3524-3536, December 2006.

[11] Y. Liu, X. Xia and H. Zhang, "Distributed Space-Time Coding for Full-Duplex Asynchronous Cooperative Communications," in IEEE Transactions on Wireless Communications, vol. 11, no. 7, pp. 2680-2688, 2012.

[12] H. Ochiai, P. Mitran and V. Tarokh, "Variable-Rate Two-Phase Collaborative Communication Protocols for Wireless Networks," in IEEE Transactions on Information Theory, vol. 52, no. 9, pp. 4299-4313, 2006.

[13] E. Beres and R. Adve, "Selection cooperation in multi-source cooperative networks," in IEEE Transactions on Wireless Communications, vol. 7, no. 1, pp. 118-127, 2008.

[14] K.Ho-Van, S. C. Paschalis, and F. Steven "Underlay cooperative cognitive networks with imperfect Nakagami-m fading channel information and strict transmit power constraint: interference statistics and outage probability analysis," Journal of Communications and Networks, vol. 16, no. 1, pp. 10-17, 2014.

[15] X. Wang and J. Li, "Network Coding Aware Cooperative MAC Protocol for Wireless Ad Hoc Networks," in IEEE Transactions on Parallel and Distributed Systems, vol. 25, no. 1, pp. 167-179, 2014.

[16] JJ. Alonso-Zárate, E. Kartsakli, Ch. Verikoukis and L. Alonso, "Persistent RCSMA: A MAC Protocol for a Distributed Cooperative ARQ Scheme in Wireless Networks," EURASIP J. Advances in Signal Processing, vol. 2008, pp. 1-13, 2008.

[17] H. Adam, W. Elmenreich, C. Bettstetter and S. M. Senouci, "CoRe-MAC: A MAC-Protocol for Cooperative Relaying in Wireless Networks," GLOBECOM 2009 - 2009 IEEE Global Telecommunications Conference, Honolulu, HI, pp. 1-6, 2009.

[18] Jianfeng Wang, Hongqiang Zhai, Yuguang Fang, J. M. Shea and Dapeng Wu, "OMAR: Utilizing Multiuser Diversity in Wireless Ad Hoc Networks," in IEEE Transactions on Mobile Computing, vol. 5, no. 12, pp. 1764-1779, 2006.

[19] A. S. Ibrahim, Z. Han and K. J. R. Liu, "Distributed energy-efficient cooperative routing in wireless networks," in IEEE Transactions on Wireless Communications, vol. 7, no. 10, pp. 3930-3941, 2008.

[20] Hao Zhu and Guohong Cao, "rDCF: A Relay-Enabled Medium Access Control Protocol for Wireless Ad Hoc Networks," in IEEE Transactions on Mobile Computing, vol. 5, no. 9, pp. 1201-1214, 2006.

[21] T. A. Khalaf and H. Mohammed, "Bit error rate performance analysis of AC-MAP in multiple input single output wireless relay network," EURASIP Journal on Wireless Communications and Networking, vol. 1, pp. 1-13, 2020.

[22] M. Dakhel and S. Hassan, "Stable High-throughput Energy Efficiency Reliable Protocol for Wireless Body Area Networks," In 2019 2nd Scientific Conference of Computer Sciences (SCCS), pp. 32-37, 2019.

[23] D. Jiang, L. Wenpan, and L. Haibin,"An energy-efficient cooperative multicast routing in multi-hop wireless networks for smart medical applications," Neurocomputing, no. 220, pp. 160-169, 2017.

[24] A. H. Radie and A. A. Thabit, "Energy Harvesting based System: Toward Outage Probability Minimizing of WBSN," In 2019 2nd International Conference on Engineering Technology and its Applications (IICETA), pp. 89-93, 2019.

[25] A. S. Al-Khaleefa, M. R. Ahmad, R. C. Muniyandi, R. F. Malik, and A. A. M. Isa. "Optimized authentication for wireless body area network." Journal of Telecommunication, Electronic and Computer Engineering (JTEC), vol. 10, no. 2, pp. 137-142, 2018.

[26] W. Taylor, S. A. Shah, K. Dashtipour, A. Zahid, Q. H. Abbasi and M. A. Imran, "An Intelligent Non-Invasive Real-Time Human Activity Recognition System for Next-Generation Healthcare," Sensors, vol. 20, no. 9, pp. 26-53, 2020

[27] B. Manickavasagam and B. Amutha, "Analysis of efficient unmanned aerial vehicles to handle medical emergency data transmission surveillance system by using wireless body area network," Computer Communications, vol. 152, pp. 19-33, 2020. 
[28] J. G. Proakis, “Communication System Engineering,” Chapter 9, p. 600, 2002.

[29] A. Bletsas, A. Khisti, D. P. Reed and A. Lippman, "A simple Cooperative diversity method based on network path selection," in IEEE Journal on Selected Areas in Communications, vol. 24, no. 3, pp. 659-672, 2006

[30] M. K. Simon and M.-S. Alouini, "Digital Communication over Fading Channels: A Unified Approach to Performance Analysis," New York: Wiley, 2000

[31] K. Noh, E. Serpedin and B. Suter, "Optimum Cooperation of the Cooperative Coding Scheme for Frequency Division HalfDuplex Relay Channels," in IEEE Transactions on Wireless Communications, vol. 6, no. 5, pp. 1654-1658, 2007.

\section{BIOGRAPHIES OF AUTHORS}

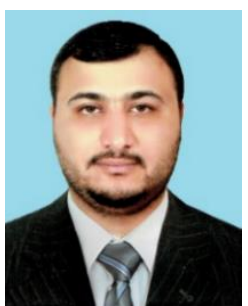

Ahmed Allawy Alawady received his bachelor degree (B.Eng.) in electrical engineering from University of Kufa, Iraq in 2007. He received master degree (M.Eng.) in electrical power system from (SHUATS), India in 2012. He is lecturer at Department of Computer Technical Engineering, Collage of Technical Engineering, The Islamic University, Iraq since 2007. Currently he is pursuing his Ph.D. degree in Universiti Tun Hussein Onn Malaysia (UTHM). His research is on motors faults detection using frequency response analysis (FRA) technique.

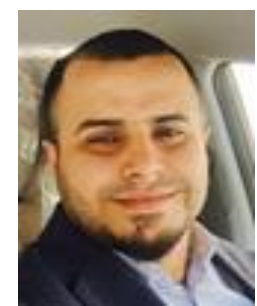

Ahmed Alkhayyat received the B.Sc. degree in electrical engineering from AL KUFA University, Najaf, Iraq, in 2007, the M.Sc. degree from the Dehradun Institute of Technology, Dehradun, India, in 2010, and Ph.D from the university of Cankaya, Ankara, Turkey, in 2015. He contributed in organizing several IEEE conferences, workshop, and special sessions. He is currently a dean of international relationship and manager of the world ranking in the Islamic university, Najaf, Iraq. To serve my community, I acted as a reviewer for several journals and conferences. His research interests include IoT health-care based, network coding, cognitive radio, efficient-energy routing algorithms and efficient-energy MAC protocol in cooperative wireless networks and wireless local area networks, as well as cross-layer designing for selforganized network.

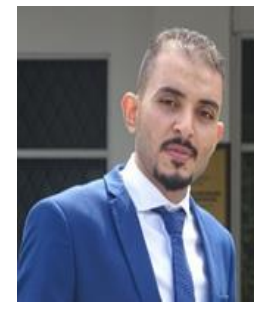

Mohammed Ahmed Jubair received the B.Sc. degree in computer engineering from Al-Marrif University, Iraq, in 2012, and the M.Sc. degree from the Computer Science Networks Department, Universiti Kebangsaan Malaysia (UKM), in 2017. He is currently pursuing the Ph.D. degree with the Faculty of Computer Science and information technology, Universiti Tun Hussen onn Malaysia (UTHM).

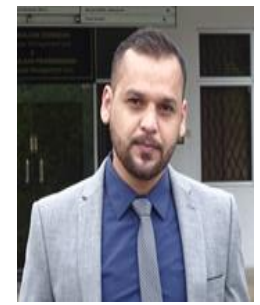

Mustafa Hamid Hassan received the B.Sc. degree in Computer engineering from Al-Marrif University, Iraq, in 2012, and the M.Sc. degree from the Computer Science Networks Department, Universiti Kebangsaan Malaysia (UKM), in 2017. He is currently pursuing the Ph.D. degree with the Faculty of Computer Science and Information Technology, Universiti Tun Hussen onn Malaysia (UTHM).

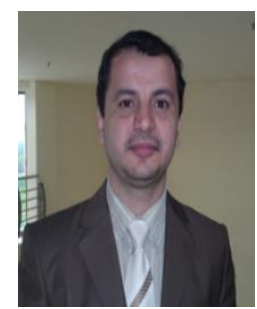

Salama A. Mostafa received the B.Sc. degree in computer science from the University of Mosul, Iraq, in 2003, and the M.Sc. and PhD degrees in information technology from Universiti Tenaga Nasional, Malaysia, in 2011 and 2016, respectively. He is currently a Lecturer at Universiti Tun Hussein Onn Malaysia, Malaysia. His research interests are in the areas of software engineering, artificial intelligence and their integration including software agents and intelligent autonomous systems. 